\title{
Minimally invasive lung resection after induction therapy: Is there evidence?
}

\author{
Bernard J. Park, MD
}

Feature Editor Note-Minimally invasive lobectomy should arguably be considered the standard surgical approach to stage I non-small cell lung cancer. There is strong evidence to support this assertion. For more than a decade now, surgeons have recognized that the benefits of thoracoscopic and robotic lobectomy could be extended to patients with locally advanced tumors, including those undergoing induction therapies. Importantly, in the current era of immunotherapy and molecular targeted therapy, thoracic surgical practices have become enriched for these patients. Whereas evidence supporting minimally invasive lobectomy after induction therapy is accumulating, it is not as robust as that which is available for early-stage disease. In this Feature Expert Opinion article, available evidence for minimally invasive lobectomy after induction therapy is contextualized by an author whose experience in this field has largely guided its direction. The reader will find a balanced interpretation of this body of literature that that will promote their safe adoption of minimally invasive lobectomy for locally advanced non-small cell lung cancer.

\section{Bryan M. Burt, MD}

Minimally invasive surgical (MIS) techniques (video-assisted thoracoscopic surgery [VATS] and robotic) for the primary treatment of early-stage lung cancer are known to be feasible and effective. Moreover, compared with the historical standard (thoracotomy), MIS approaches have advantages, including reduced complication rates, shorter length of hospitalization, decreased early postoperative pain, and quicker recovery. In the initial published experiences with both VATS and robotics, patients with locally advanced disease (larger tumors, invasion of adjacent structures, hilar and mediastinal nodal metastases) were

\footnotetext{
From the Thoracic Service, Department of Surgery, Memorial Sloan-Kettering Cancer Center, New York, NY.

This work was supported in part by National Institutes of Health/National Cancer Institute Cancer Center Support Grant P30 CA008748.

Received for publication April 8, 2020; accepted for publication July 8, 2021; available ahead of print Aug 31, 2021.

Address for reprints: Bernard J. Park, MD, Thoracic Service, Department of Surgery,

Memorial Sloan-Kettering Cancer Center, 1275 York Ave, Room C-879, New

York, NY 10065 (E-mail: parkb@mskcc.org).

JTCVS Open 2021;8:585-7

2666-2736

Copyright (C) 2021 The Author(s). Published by Elsevier Inc. on behalf of The American Association for Thoracic Surgery. This is an open access article under the CC BY-NC-ND license (http://creativecommons.org/licenses/by-nc-nd/4.0/).

https://doi.org/10.1016/j.xjon.2021.07.038
}
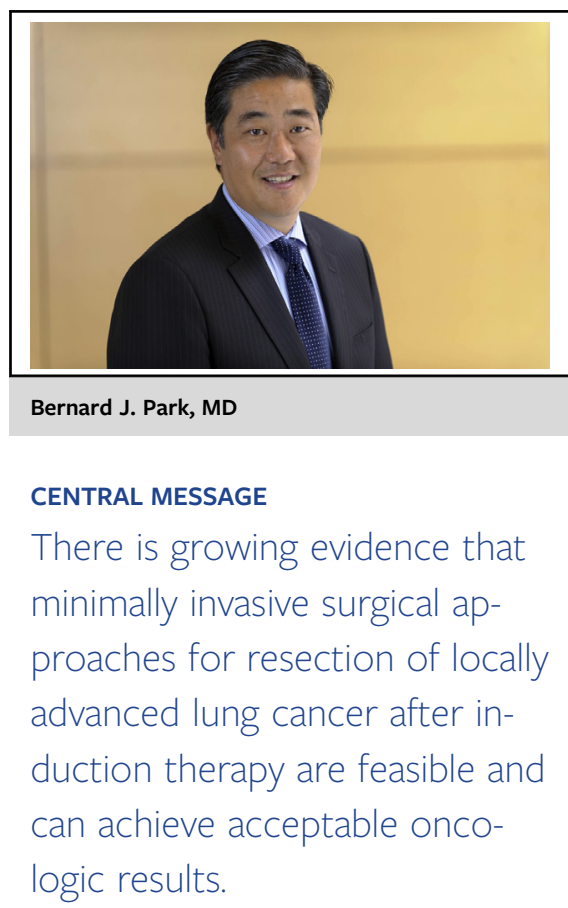

generally excluded. In particular, receipt of induction therapy was a relative contraindication to approaching resection by MIS. However, as experience and technology have evolved, it has become evident that VATS and robotics are being increasingly used in patients who have undergone induction therapy. The questions are whether there is evidence to support this trend and what considerations are necessary for successful implementation.

Unfortunately, there are precious few randomized, prospective trials comparing MIS with thoracotomy for the treatment of lung cancer, and with respect to the role of MIS after induction therapy there are virtually none. Currently, one of the largest randomized trials comparing VATS with open lobectomy for lung cancer is the ongoing Video-assisted thoracoscopic lobectomy versus conventional open lobectomy for lung cancer (the VIOLET study). It will compare the effectiveness, cost-effectiveness, and acceptability of VATS versus open lobectomy for lung cancer with the primary outcome being self-reported physical functioning 5 weeks after randomization and among the secondary outcomes the oncologic outcomes. Unfortunately, this study will not include patients undergoing induction therapy but nonetheless will be an important addition to the literature. There are several high-quality, retrospective series both cohort and case-control studies that have shed 
light on feasibility and oncologic efficacy of MIS following induction. One of the earliest experiences came from Duke University by Petersen and colleagues, ${ }^{2}$ who reported on 97 consecutive patients (85 thoracotomy, 12 VATS) who had induction chemotherapy and lobectomy. Despite the retrospective nature of the study, the groups had similar perioperative characteristics except that the open patients had a greater proportion of clinical stage IIIA and larger tumors. The patients who received VATS had significantly shorter chest tube duration ( 2 days less), decreased length of stay (1.5 days less), comparable morbidity, mortality, and similar survival.

A number of subsequent feasibility studies have been reported in the subsequent decade. Most of these studies are uncontrolled, single-institution case series. Huang and coauthors $^{3}$ from China looked at the feasibility of using VATS after induction therapy in 43 consecutive patients with clinical stage IIA to IIIB disease. Preoperative treatment consisting of targeted therapy (5), chemotherapy (23), and chemoradiotherapy (15). Procedures included lobectomy, bilobectomy, pneumonectomy, and sleeve resections with acceptable operative times, postoperative morbidity/mortality $(9.5 \% / 2.4 \%)$, and a $95 \% \mathrm{R} 0$ resection rate. There were $7(16.7 \%)$ conversions to "hybrid" thoracotomy. With a median follow-up time of 21 months, the overall survival was 33 months, with 1-year, 2-year, and 3 -year survivals of $94 \%, 79 \%$, and $65 \%$, respectively. A similar series from China by Yang and $\mathrm{Zhai}^{4}$ evaluated the feasibility of a uniportal VATS approach for management of locally advanced lung cancer. They reported on 29 consecutive patients, all of whom received 2 cycles of induction chemotherapy followed by attempted uniportal VATS lobectomy. Two patients underwent multiport VATS, and only 1 patient $(3.4 \%)$ was converted to thoracotomy. Overall complication rate $(17.2 \%)$ and mortality were low $(0 \%)$.

There have been a few case-control studies comparing MIS approaches with thoracotomy following induction treatment. A retrospective series from Korea by Jeon and colleagues ${ }^{5}$ looked specifically at 35 patients with stage IIIA (N2) who underwent preoperative chemoradiation followed by VATS (17) or thoracotomy (18) for complete resection. This, unfortunately, was not an intent-to-treat analysis, and the 5 of the $22(23 \%)$ patients who were converted were analyzed in the thoracotomy group. Even with this somewhat-flawed methodology, there were no differences between groups in terms of perioperative outcome. With a median follow-up of 36 months' survival, both overall and disease-free were comparable. A larger series looking at greater longitudinal and long-term survival results is an update of Duke's cumulative experience by Yang and colleagues in which they analyzed a total of 272 patients treated by open (203) or VATS lobectomy (69) for locally advanced disease. ${ }^{6}$ The patients who received VATS had greater clinical stage, were older, and had a greater incidence of coronary disease and chronic obstructive pulmonary disease, whereas patients who underwent thoracotomy had a greater rate of induction radiotherapy. With a conversion rate of $10 \%$, patients who received VATS had significantly lower rates of postoperative pneumonia and shorter chest tube duration and hospital stay. After a median follow-up of 24 months, overall and disease-free survival were equivalent in both groups.

Another large case-control experience and the only one to include patients treated by thoracotomy, VATS, and robotic approaches comes from Memorial Sloan-Kettering Cancer Center. ${ }^{7}$ Over a period of 11 years and a median follow-up of 40 months, 428 patients (397 open, 17 robotic, 14 VATS) underwent lobectomy following induction therapy. There were no major differences in preoperative characteristics between groups, and virtually all patients underwent complete (R0) resection (94\% open, 97\% MIS). Conversion rate was $26 \%$, and consistent with previous series the MIS group had shorter length of hospitalization. Survival was comparable between surgical approaches. A similar study reported by Kamel and colleagues $^{8}$ at Cornell performed a 1:2 ratio propensitymatched analysis of 114 patients (74 open, 40 VATS) who had lobectomy following induction in an effort to minimize selection bias. With a conversion rate of $12.5 \%$, thoracoscopy was associated with less blood loss, chest tube duration, and length of stay. There were trends toward superior disease-free survival and freedom from recurrence in the VATS group, leading the authors to suggest that it is the preferred surgical approach. The sole factor associated with improved outcome was the clinical $\mathrm{N}$ status of the patients, both on univariable and multivariate analysis.

Lastly, it is worth nothing that with the publication of the PACIFIC trial ${ }^{9}$ that established the role of immune checkpoint inhibitors in the multimodality treatment of locally advanced lung cancer, there has been a tidal wave of trials adding immune checkpoint inhibitors to induction regimens. Most have yet to be published yet, but most include patients undergoing both MIS and open resection. Bott and colleagues ${ }^{10}$ from Memorial Sloan-Kettering Cancer Center and Johns Hopkins reported the surgical results of lung resection after 2 cycles neoadjuvant nivolumab. Thirteen $(65 \%)$ of the patients had an attempt at VATS or robotic resection, and 7 (54\%) required nonurgent conversion, all for hilar fibrosis and/or bulky nodal disease. Of the 20 patients who underwent successful resection, a total of $70 \%$ had open surgery. The entire group did well, however, with a median length of stay of 4 days and no operative mortality. Similarly, Shu and colleagues ${ }^{11}$ published results of a multicenter, single-arm phase 2 trial of induction atezolizumab, nab-paclitaxel, and carboplatin followed by surgery for stage IB-IIIA non-small cell lung cancer. Of the 29 patients who went to the operating room, 26 had complete 
(R0) resection, and of those, $12(46 \%)$ had VATS whereas the remainder $(14,54 \%)$ had thoracotomy. It is unknown how many were converted to thoracotomy. Once again, median length of stay was 4 days, and there was 1 perioperative death $(3 \%)$.

There are several conclusions that are evident from reviewing the available studies. First, there is limited highlevel evidence to be able to strongly advocate that MIS should be the standard surgical approach following induction therapy for locally advanced lung cancer. The use of VATS and robotic approaches for pulmonary resection continues to increase, but overall, there is still a high proportion of all cases done by thoracotomy. Given the generally increased complexity and issues associated with postinduction resections, it is not likely that MIS approaches will be universally adopted or feasible. However, depending on the experience and comfort level of the surgeon, most patients can be offered an attempt at MIS resection, and conversion in this setting is not a failure. Situations that are highly likely to necessitate thoracotomy include bulky tumors with primary or hilar nodal disease adherent to the hilar vasculature. Clinical scenarios that require arterioplasty or vascular sleeve resection should only be approached by MIS in the most experienced hands or only by open surgery.

Second, based on good retrospective cohort and casecontrol studies, one can conclude that, in appropriately selected patients, use of MIS techniques can result in improved perioperative outcomes and equivalent oncologic outcomes to standard thoracotomy. It is clear that careful perioperative planning and strict conduct of the MIS procedure can lead to a high rate of complete resection and a low number of conversions. A video-assisted approach allows for execution of the same critical operative steps as the open approach and does not require development of a unique procedure. In some instances, use of video technology may facilitate certain portions. The telerobotic platform, in my experience, with its magnified, binocular vision and articulated instrumentation makes both complete lymph node dissection and postinduction hilar dissection frequently easier than VATS or open.

Third, conversion to thoracotomy is not a failure and should be anticipated in up to $25 \%$ of cases. The most important goals of the surgical management of locally advanced lung cancer are patient safety and oncologic efficacy. Elective conversion to avoid catastrophic injuries or to preserve oncologic principles should be embraced, and urgent thoracotomy for hemorrhage or other critical situations, while unavoidable, ought to be an uncommon experience. If one adheres to those principles, there is no reason why the vast majority of these patients can at least be offered or initiated with minimally invasive approaches.

\section{Conflict of Interest Statement}

Dr Park is a proctor and speaker for Intuitive Surgical. He has received consulting fees from COTA and Astra Zeneca.

The Journal policy requires editors and reviewers to disclose conflicts of interest and to decline handling or reviewing manuscripts for which they may have a conflict of interest. The editors and reviewers of this article have no conflicts of interest.

\section{References}

1. Lim E, Batchelor T, Shackcloth M, Dunning J, McGonigle N, Brush T, et al Study protocol for VIdeo assisted thoracoscopic lobectomy versus conventional Open LobEcTomy for lung cancer, a UK multicentre randomized controlled trial with an internal pilot (the VIOLET study). BMJ Open. 2019;9:e029507.

2. Petersen RP, Pham D, Toloza EM, Burfeind WR, Harpole DH Jr, Hanish SI, et al. Thoracoscopic lobectomy: a safe and effective strategy for patients receiving induction therapy for non-small cell lung cancer. Ann Thorac Surg. 2006;82: 214-8.

3. Huang J, Xu X, Chen H, Yin W, Shao W, Xiong X, et al. Feasibility of complete video-assisted thoracoscopic surgery following neoadjuvant therapy for locally advanced non-small cell lung cancer. $J$ Thorac Dis. 2013;5(Suppl 3):S267-73.

4. Yang Z, Zai C. Uniportal video-assisted thoracoscopic surgery following neoadjuvant chemotherapy for locally-advanced lung cancer. J Cardiothorac Surg. 2018;13:33-8.

5. Jeon YJ, Choi YS, Lee KJ, Lee SH, Pyo H, Choi JY. Outcomes of pulmonary resection and mediastinal node dissection by video-assisted thoracoscopic surgery following neoadjuvant chemoradiation therapy for stage IIIA N2 nonsmall cell lung cancer. Korean J Thorac Cardiovasc Surg. 2018;51:29-34.

6. Yang CJ, Meyerhoff RR, Mayne NR, Singhapricha T, Toomey CB, Speicher PJ, et al. Long-term survival following open versus thoracoscopic lobectomy after preoperative chemotherapy for non-small cell lung cancer. Eur J Cardiothorac Surg. 2016;49:1615-23.

7. Park BJ, Yang HX, Woo KM, Sima CS. Minimally invasive (robotic assisted thoracic surgery and video-assisted thoracic surgery) lobectomy for the treatment of locally advanced non-small cell lung cancer. J Thorac Dis. 2016;8(Suppl 4): S406-13.

8. Kamel MK, Nasar A, Stiles BM, Altorki NK, Port JL. Video-assisted thoracoscopic lobectomy is the preferred approach following induction chemotherapy J Laparoendosc Adv Surg Tech A. 2017;27:495-500.

9. Antonia SJ, Villegas A, Daniel D, Vicente D, Murakami S, Hui R, et al. Durvalumab after chemoradiotherapy in stage III non-small cell lung cancer. $N$ Engl J Med. 2017;377:1919-29.

10. Bott MJ, Yang SC, Park BJ, Adusumilli PS, Rusch VW, Isbell JM, et al. Initial results of pulmonary resection after neoadjuvant nivolumab in patients with resectable non-small cell lung cancer. J Thorac Cardiovasc Surg. 2019;158:269-76.

11. Shu CA, Gainor JF, Awad MM, Chiuzan C, Grigg CM, Pabani A, et al. Neoadjuvant atezolizumab and chemotherapy in patients with resectable non-smallcell lung cancer; an open-label, multicentre, single-arm, phase 2 trial. Lancet Oncol. 2020;21:786-95.

Key Words: induction therapy, neoadjuvant, VATS, thoracoscopic, robotic surgery, minimally invasive surgery (MIS) 